\title{
Historia
}

\section{La enfermería como "recurso": el proceso de profesiona- lización de la enfermería en la década del '70, entre las políticas de Estado y el modelo Médico- hegemónico}

\author{
Nursing as a "resource": the profesionalization process of nursing in \\ the 70's, between State policies and the medical-hegemonic model.
}

\section{A Enfermagem, como um "recurso": o processo de profissionalização da enfermagem na década de ' 70 , entre as políticas de Estado e o mode- lo médico- hegemônico}

Esteban Justo

Facultad de Ciencias de la Salud, Universidad Nacional de Mar del Plata

Cómo citar este artículo en edición digital: Justo, E. (2014) La enfermería como "recurso": el proceso de profesionalización de la enfermería en la década del '70, entre las políticas de Estado y el modelo Médico- hegemónico. Cultura de los Cuidados (Edición digital) 18, 39. Disponible en: http://dx.doi.org/10.7184/cuid.2014.39.05>

Correspondencia: Ituzaingo 3520, Mar del Plata, Argentina. Codigo Postal 7600

Correo electrónico: "estebanjusto@hotmail.com"

Recibido: 02/03/2014; Aceptado: 17/05/2014

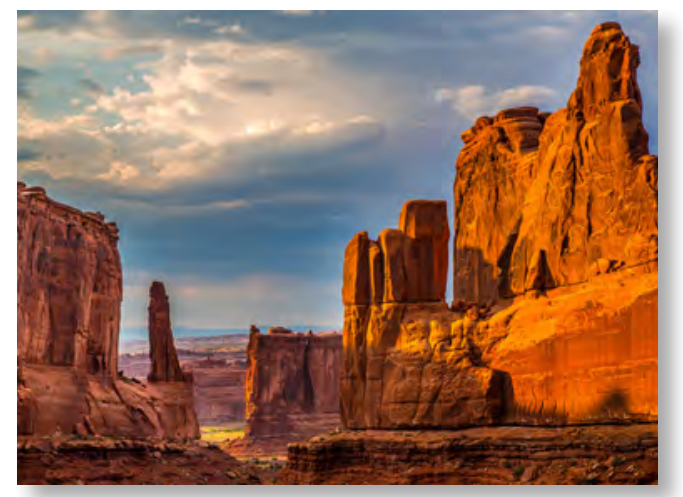

\section{ABSTRACT}

This research is an attempt to portray the changes in nursing professional practice, placed in the 70's at the INE "Juan H Jara”, as well as the different changes in State policies and it's relationship with the medical corporation.

We analyzed the nursing discourse and compared it with documents of this time, in an attempt to shed light on the dynamics and structure of the social processes of reproduction, that had a gradual "invisibility" effect on nursing practice. Nursing has been considered as a "resource" by doctors, much like medical supplies, so that they could "stock up" or dispose of, according to momentary needs, this "instrumentalisation" is relatable to difficulties for the development of critical analysis and collective processes of resistanse to professional alienation.

Keywords: Nursing history - Hegemonic Medical Model - Social capital - Crisis.

\section{RESUMO}

A pesquisa procura dar conta das mudanças que ocorrem com a prática profissional de enfermagem do INE “Juan H Jara” da década de 70 , as várias mudanças do Estado nacional 
e suas relações com a medical corporation. Confrontando o discurso enfermeira com os documentos e dados do tempo, temos tentado interpretar aquilo que foi a estrutura e dinâmica dos processos de reprodução social, que teve como resultado a progressiva "invisibilidade" das práticas de enfermagem. Enfermagem foi considerado como um "recurso" pelos médicos, o que não é muito diferente de outros insumos, que poderia construir e desfazer segundo as necessidades do momento, esta instrumentalização está relacionada com as dificuldades no desenvolvimento de competências de análise crítica e resistência colectiva para o alienante dos processos dentro da profissão.

Palavras -chave: História da emfermagem - Modelo medico-hegemônico - Capital social - Crise.

\section{RESUMEN}

La investigación que nos planteamos busca dar cuenta de las transformaciones acaecidas a la práctica profesional de enfermería en el INE "Juan H Jara” en la década de los 70, los distintos cambios del Estado nacional y sus relaciones con la corporación médica. Cotejando el discurso enfermero con documentos y datos de la época, intentamos interpretar cuál fue la dinámica y estructura de los procesos de reproducción social, que tuvieron como resultante la gradual "invisibilización" de las prácticas de enfermería. La enfermería fue considerada como un "recurso" por los médicos, no muy distinto de otros insumos, que podían construir y deshacer según las necesidades coyunturales, esta instrumentalización se relaciona con las dificultades para el desarrollo de la capacidad de análisis crítico y la resistencia colectiva a los procesos alienantes dentro de la profesión.
Palabras clave: Historia de la enfermería Modelo Medico Hegemónico - Capital social - Crisis.

\section{INTRODUCCIÓN}

La investigación que nos planteamos busca dar cuenta de las transformaciones acaecidas a la práctica profesional de enfermería, en el Instituto Nacional de Epidemiología "Juan $\mathrm{H}$. Jara” en Mar del Plata, Argentina (en adelante INE).

Tomamos como premisa la teoría de que el perfil de enfermería surgió en nuestras instituciones, condicionado por una disputa del "campo de fuerzas" de la salud, con otro grupo profesional que históricamente tiene un mayor grado de organización y ocupa un status hegemónico: los médicos. Colliere (1986) plantea que el desarrollo histórico de los saberes de la enfermería están íntimamente vinculados a la "invisibilización" de la cultura de la mujer por parte del patriarcado, primero a través de la Iglesia y luego en la relación de auxiliaridad establecida con los médicos, en lo que dio a llamar "la doble filiación conventual y medica", y su lucha por romper ese yugo.

Enfocándonos en la historia de la enfermería argentina, Morrone explica como los procesos de profesionalización de la enfermería encontraban una enconada oposición por parte de grupos de poder, como la Iglesia $\mathrm{Ca}$ tólica, que sostenían un modelo de salud "de beneficencia” (Morrone, 2012). Esta oposición se manifestó primero frente a la Escuela de Cecilia Grierson con la primera secularización de la salud bajo el impulso del higienismo, y luego en las expresiones más avanzadas del desarrollo de una "nueva enfermería", que la autora sitúa en la escuela de enfermería de la Fundación Eva Perón. Esta "nueva enferme- 
ría” expresaba una paradigma de enfermera con autonomía de la figura de los médicos, profesional y técnicamente solvente, contradiciendo los valores y la estética de subalternidad impuestas al genero femenino de la época. Vemos aquí un enfrentamiento de proyectos que tienen que ver con contenidos de clase $y$ de género, y consideramos que se puede enriquecer esta perspectiva analizando los cambios del Estado nacional y sus relaciones con la corporación médica, ya que esta dinámica es la que le determina al sistema de salud en sus rasgos principales: entendemos como Modelo Medico-Hegemónico el monopolio del capital, tanto simbólico como material, que detenta esta corporación en campo de la salud. El Estado actúa como socio, regulando o desregulando, avanzando o retrocediendo, ejerciendo el poder en una alianza estrecha, para resguardar sus ganancias y su posición hegemónica. En estos procesos se abren y cierran establecimientos, se adoptan modalidades de trabajo y se desechan otras, siempre supeditadas a la lógica capitalista, donde la salud es principalmente una mercancía y no un derecho.

Como en la mayoría de las relaciones de poder en la sociedad de clases, la dominación sobre los enfermeros en tanto clase subalterna, suele tener un cariz despiadado, y muchas veces termina instrumentalizando a los sujetos. Esto queda expresado claramente en el discurso enfermero del INE: “...Acá siempre hubo esa cultura de 'úsemos a enfermería' para sacar adelante cosas que después era rédito de los médicos, eso termino agotando a los profesionales de enfermería" (L.S., entrevista personal, Noviembre 2013). Es necesario reconstruir los pormenores de este proceso, analizando el ejercicio del poder en la institución, y contextualizándolo en los vaivenes del modelo Estatal.

\section{MÉTODO}

Se trata de un estudio historiográfico, inspirado por un trabajo previo en el marco de la Licenciatura en Enfermería de la Universidad Nacional de Mar del Plata, Argentina, donde el autor esta finalizando su tesis de grado.

El punto de partida de toda historiografia es la problematizacion de lo actual a partir de una situacion que se configure como posibilidad de realizar una investigación historica. El campo de la historia es un campo de posibilidades que debe trabajarse con los "ahoras" a ser investigados (Padilha MICS, Borenstein MS. 2005). Los testimonios recogidos de los jovenes profesionales de enfermería evidencian la sensación de no poder desarrollarse profesionalmente, de no poder desempeñar una actividad enriquecedora de la práctica, que produce una profunda desconexión con el colectivo profesional, y una desvalorización reflejando la crisis actual de la enfermería en el INE.: "después de muchos años de trabajo, te quema el cerebro que los compañeros (enfermeros) nos serruchemos los unos a otros el piso, entonces llega un momento que no te importa lo que le pasa al otro", "entonces o te dejas estar, o trabajas para vos, solamente para vos, y no en función del colectivo enfermero, que debería ser como realmente se trabaja en enfermería” (L.E. entrevista personal, Octubre 2013)

Buscando el origen de esta crisis en la hipotesis que era consecuencia de las politicas neoliberales de los '90s, es que nos encontramos en la necesidad de "darle voz" a los hechos acaecidos en un periodo anterior. Para ello recurrimos a entrevistas en modalidad de "historia de vida" como fuentes primarias, efectuadas a enfermeras y personal que cumplia funciones en la institución, y documentos escritos como periódicos, Memorias institucionales e Historias clínicas. 
Las fuentes secundarias fueron las obras que aportaron elementos para la revisión critica y sistemática de las fuentes primarias, en un intento por interpretar cuál fue la dinámica y estructura de los procesos de reproducción social, que tuvieron como resultante la gradual “invisibilización” de las prácticas de enfermería, mientras que se reforzaba el "capital social" de otros colectivos profesionales, en perjuicio de aquellas.

\section{DESARROLLO DEL TEMA}

En un primer momento la enfermería en el INE tiene características de oficio más que de profesión, propio de un modelo de salud orientado hacia la "beneficencia", donde las primeras "enfermeras" eran empíricas y fueron mucamas entrenadas por las monjas que aún residían y cumplieron funciones en enfermería hasta entrada la década del '70, hemos podido constatar registros del año ' 71 donde figuran en la hoja de evolución de enfermería la firma de la "Hermana Bernardita" que cumplía horario en la Sala de Mujeres de mañana.

Analizando los documentos de principios de esta década, encontramos evidencias de un ida y vuelta entre dos proyectos antagónicos de institución: Una de corte más asistencial y de orientación a la salud comunitaria, con rasgos nacionalistas, que en el INE se expreso en el modelo 1968-1973 y otra más técnica, supraestructural y burocrática, cuyos cuadros técnicos están vinculados con los organismos internacionales, que es la contracara de la anterior e identificamos con el proyecto de 19711976.

Estas dos recetas están vinculadas con una mayor o menor apertura del Estado a la influencia de los capitales internacionales, el desarrollo del capitalismo en la Argentina ha estado vinculado a distintos modelos, muchas veces expresados en concepciones y proyectos antagónicos de nuestro país (Iriart, Nervi et al ,1994). En el INE esta lucha decanto en dos posturas con lineamientos claros en cuanto a las funciones de la institución en el sistema de salud, y los efectos que tiene esto sobre la enfermería son contundentes. Empezaremos por analizar el primero de estos modelos.

A principios de la década del 70, aprovechando las concepciones de modernización del Estado desarrollista, el entonces director Munaffo Daucia da refugio en el INE a un grupo de médicos que estaban peleados con el Centro Medico y las clínicas existentes en la ciudad, y que luego pasarían a fundar el Hospital de Comunidad, para ejercer como médicos de consultorio (E.C., entrevista personal, Octubre 2013). Esto cerraba la transición desde el modelo de atención de "beneficencia" en el INE, ya que la institución sería la sede de un ambicioso proyecto piloto: el "Centro Nacional de Lucha Antituberculosa de Mar del Plata”, cuya misión era la de poner en marcha un plan de salud Nacional destinado a erradicar la Tuberculosis coordinando todos los servicios de salud, con una jurisdicción que se extendía hacia el sur del país hasta Comahue y la Patagonia. La institución adopta características de tecnificación, a principio de junio de 1968 habían inaugurado unos modernos consultorios externos que por sus "comodidades de espera y circulación, y la vinculación rápida con Laboratorios y Sala de Rayos mejorando sensiblemente la atención brindada" (Memorias INE 1968 pag. 25).

Se postula una concepción interdisciplinaria del trabajo de atención: "una vez rotulado un paciente nuevo con tuberculosis por haber obtenido la confirmación bacteriológica, su historia clínica es complementada mediante los estudios pertinentes, por el servicio social, 
el servicio de psicología y psiquiatría y el servicio de enfermería y su discusión es llevada al primer sábado de la semana en que se finaliza el estudio del caso." (Memorias INE 1968 pag. 25)

Si bien este esquema sorprende por lo integrado y avanzado en sus concepciones, las entrevistas reflejan que las relaciones institucionales respondían en los hechos a un esquema verticalista y jerárquico: "Cuando estaba $\mathrm{Mu}$ naffo (el Director) era bien militar todo acá, el era capitán de la Marina. Si no le gustabas... fuistes! " , "Cada servicio tenia su color de uniforme diferenciado, eso era para saber a simple vista quien andaba dando vueltas, por fuera de su lugar de trabajo"(S.P., entrevista personal, Noviembre 2013).

En este clima de disciplina la enfermería ocupaba un rol central en las actividades, si bien marcado por las limitaciones del paradigma auxiliar-asistencialista, esto le exigió una integración como colectivo y un compromiso con sus tareas que fue destacado, el desarrollo de las enfermeras como "recurso" permitió establecer hasta un Departamento de Enfermería, con un "local adecuado para instalar las dependencias de la Jefatura del Departamento, lugar de reuniones, ambiente de trabajo administrativo, y como anexo, dormitorio para la Supervisora de Guardia.”

Se inicio una modalidad de tratamiento "intermitente", que quedo a cargo del personal de Enfermería "con estrecha coordinación con el Servicio Social”. El tratamiento se realizaba suministrando las drogas directamente en el domicilio del paciente, con lo cual algunas de las enfermeras trabajaban en un perfil de salud comunitaria: "El Departamento de Enfermería asumió funciones irremplazables en lo que se refiere a la atención de los enfermos, que se extendieron hasta la atención en el hogar y se prolongo hasta la educación del paciente y sus familiares en cuanto hace a la prevención de las enfermedades y la protección de la salud." (Memoria del INE año 1968 pag. 28). La tarea de enfermería extramuros abría la posibilidad de un ejercicio autónomo de la profesión.

Estas actividades exigían funciones docentes, de supervisión de prácticas, además motivaban una formación permanente como la concurrencia a los ateneos médicos. En el mes de Noviembre, por ejemplo "con motivo de celebrarse el Día de la Enfermería, se llevaron a cabo dos Seminarios: uno sobre acción del Centro Nacional de Lucha Antituberculosa en la Comunidad y el otro sobre Actualización de enfermería en la tuberculosis."(Memoria del INE año 1968 pag. 42). Otra referencia indica los procesos de jerarquización y especialización que se iban dando hacia adentro del colectivo enfermero: "También se llevo a cabo el Adiestramiento en Servicio de Enfermería a cargo de la Jefe del Departamento Srta. Luisa G. Muller, al que concurre todo el personal de enfermeros y auxiliares del establecimiento."

Estos testimonios explican porque en el imaginario actual de las enfermeras del INE se puede identificar al periodo del "Departamento" con la época de oro de la enfermería: "el recurso de enfermería se fue achicando, O. contaba que eran como quince, y antes era 'Departamento' de enfermería” “eso se perdió y también tiene que ver con cuestiones políticas" (G.J., entrevista personal, Noviembre 2013). Se observa que la enfermería había logrado la construcción de un espacio de relativa autonomía, que si bien se encontraba en un proceso de acumulación de capital social, parafraseando a Colliere, aún estaba muy vinculada a un "rol moral", cuyo estatus dependía por el momento de la delegación técnica que en ella hacía el medico (Colliere, 1986). 


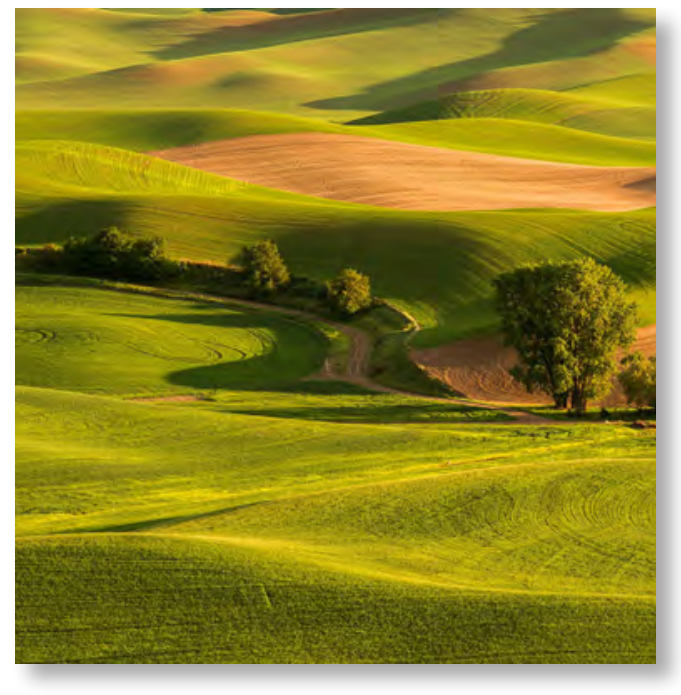

Aunque fuera como "recurso" moldeable en manos de los médicos, la actitud entusiasta, casi militante, con la que las enfermeras aprovecharon ese espacio para desarrollarse, fue sin duda un impulso importante para la profesionalización.

Todo indica que a partir de 1971, los cambios políticos y de autoridades del gobierno proyectan para el INE un rol distinto, ya no como centro asistencial, sino en la supraestructura donde se producen los conocimientos y las categorías para entender la realidad.

El Decreto 1944 del 28/06/1971 postula claramente este nuevo perfil del INE, dice: "Que resulta indispensable constituir en el país un instituto altamente especializado en el estudio de los factores ecológicos que participan en las funciones de salud y enfermedad de los grupos humanos" y en un párrafo posterior aclara "..la transformación del instituto mencionado, en un organismo del más alto nivel de la Administración Publica Nacional".

Esta misión estaba en consonancia con la vocación planificadora que asumía el Estado, como evidencia este párrafo de las Memorias de 1972: "Cualquiera sea el sistema económico político en juego, es responsabilidad de los organismos de planificación, fijar prioridades para el financiamiento de los programas y las instituciones de cada área a fin de que se pongan en marcha los recursos humanos y físicos con que cuenten."

Aquí el INE tiene la posibilidad de empezar a manejar con más fuerza un capital simbólico, como nos refiere Bourdieu(1985), la posibilidad de tener el monopolio de la 'nominación’ es la capacidad de producir la visión legitima del mundo social. Es ni más ni menos que el poder de imponer la propia producción cultural o ideológica hacia adentro del campo de la salud, con la voz del Estado, a través de la epidemiología.

Se entiende porque la nueva situación trae aparejada una reestructuración y un reacomodamiento de los recursos y prioridades institucionales. En el nuevo esquema, el rol de la enfermera ligado a lo asistencial había perdido utilidad para los médicos, que rápidamente proceden a tomar medidas.

Podemos constatar que se produce la pérdida del Departamento de Enfermería como espacio diferenciado, pasando a depender del "Departamento Servicios Técnicos Auxiliares", espacio que comparte con Farmacia, Radiología, Servicio Social y Estadística, cuya jefatura ocupaba un médico. Paralelamente hay llamativa omisión de mención tanto de las actividades como de las personas involucradas en enfermería en los distintos documentos de la época.

Asimismo la merma del personal fue notable, así como la variación en la composición del personal. De contar en 1968 con 59 personas en el Departamento, de las cuales había Supervisoras, Jefas de Sala, enfermeras y auxiliares, totalizando 22 enfermeras, en 1977 vemos que el Departamento de Servicios Técnicos contaba con 32 personas en total, de las cuales no se 
discrimina cuantas enfermeras. En el balance de actividades de 1972 no se menciona a Enfermería en ninguna parte del informe, ni siquiera cuando se refiere a las prestaciones propias del servicio como vacunación y atención en la internación. Las actividades son presentadas como un informe estadístico donde solo constan el número y porcentaje de las prestaciones. Todo sugiere que se había reducido el rol de enfermería a unas cuantas técnicas de apoyo de baja complejidad.

El esquema que se proponía anteriormente para la atención de los pacientes, donde existía una integración de las distintas disciplinas, queda muy reducido, y la enfermería brilla por su ausencia. En las historias clínicas podemos ver que a partir de 1972 ya el control de contactos de Tuberculosis lo pasan a realizar las Asistentes Sociales, y no las Enfermeras Visitadoras. El clima institucional parece haber cambiado drásticamente.

Pero parece inevitable citar la famosa frase de Marx, que la historia se repite, primero como comedia y luego como tragedia, para referirnos al capitulo final de esta lucha entre dos modelos de INE contrapuestos, tal como lo habíamos adelantado. Entre 1973 y 1975, con la segunda Presidencia de Perón, se produce una recuperación del INE abierto a la comunidad, hay una revitalización en el discurso institucional que hace eje en la internación, en la integración de una política sanitaria más amplia, con una retórica populista. Con el ascenso de Cámpora a la presidencia se produce una "breve primavera de los sectores de izquierda alineados con el Peronismo", en el cual se dan "intervenciones" que fueron de hecho tomas ejecutadas por los sectores juveniles de la militancia política, para evitar la continuidad del régimen castrense denominado "Revolución Argentina” en los organismos estatales(La Ca- pital, 2011). El INE fue uno de los establecimientos que experimento esta "intervención", que puso en funciones al Dr. Osvaldo Gatti: "A partir del 21 de Noviembre de 1973, iniciamos en el Instituto Nacional de Epidemiología una política sanitaria de puertas totalmente abiertas a la Salud de nuestro Pueblo" (Memorias INE 1974 pag. 1) "Otro objetivo de gran y fundamental importancia ha sido la de restaurar nuevamente la Sala de Internación que nunca debió dejar de funcionar en esta Institución.

Ya los pacientes no deben ser desarraigados de su núcleo familiar, ni trasladados a instituciones de otros partidos ni provincias. Con este logro social nuestra Institución reinicia su total identificación con la Salud de su Pueblo." (Memorias INE 1974 pag. 2)

El racconto de los cambios en los documentos oficiales trasluce los enfrentamientos ideológicos y de concepciones: "Por razones que no es necesario entrar a analizar, el Instituto no contó mas con la Sala de Internación, a partir del año 1972, reemplazándose el espacio físico por el Departamento Administrativo Contable. Es decir que al pretender sobretecnificarlo se le hizo perder en gran parte, esa sensibilidad a la cual me refería, que recobra nuevamente en plenitud" (Memorias INE 1975 pag. 1).

Gracias a estos cambios el INE recobraba proyección en el plano nacional delineando políticas sanitarias, por lo cual se había instalado una Radioestación que comunicaba con “Central (Buenos Aires), Noreste y Noroeste Argentino, Cuyo, Comahue, Patagonia y Pampeana. Y solucionar problemas de urgencia medica, traslado de pacientes en aviones, ambulancia, pedidos de medicamentos, etc."

De haber continuado este rumbo, posiblemente hubiera significado el resurgimiento de espacios autónomos para enfermería, sin em- 
bargo los cambios en el campo social producidos luego del año 1976, muestran que la pugna entre estos dos modelos de institución se salda de manera abrupta y definitiva.

Es a partir del golpe militar de 1976 que la tendencia en la institución de limitar las funciones sanitarias se empiezan a manifestar desembozadamente. Podemos leer en el "Enfoque General" de las Memorias: "Un análisis de la situación del instituto, determino que era necesario adecuar la institución a sus fines específicos, desarrollando en forma preeminente las actividades de Investigación Epidemiológica y Docencia, con una faz asistencial de apoyo de dichas actividades, para revertir así el predominio de la labor asistencial que la institución había tenido en los últimos años.”, el lenguaje es preciso y breve, el tono es lapidario, "El referido análisis mostró la necesidad de redimensionar el área de acción del instituto, que hasta la fecha era todo el país." Con esto se naturaliza en el INE el proyecto vencedor, dando por finalizadas las expectativas de aquellos que pretendían una acción sanitaria de "puertas abiertas".

Las Memorias de 1977 nos indican que se firmaron diversas cartas acuerdos con organismos municipales, provinciales y nacionales para externar algunas de las funciones que había asumido el INE: "se mantiene de esta forma la actitud de integración expresada el año anterior y que tiende al aprovechamiento de los recursos y el mejor cumplimiento de los objetivos de las instituciones". El neoliberalismo empieza a mostrar su lógica racionalizadora que luego desplegara con toda su fuerza en la década infame Menemista (ver Boron 2003).

La progresión de la planta de personal desde el '68 al '78 muestra un declive constante, que se agudiza abruptamente a partir del ' 76 . Entre el '76 y el ' 78 se producen 55 bajas por despido o renuncia, el doble que las bajas por toda causa en el periodo '68 a '74.

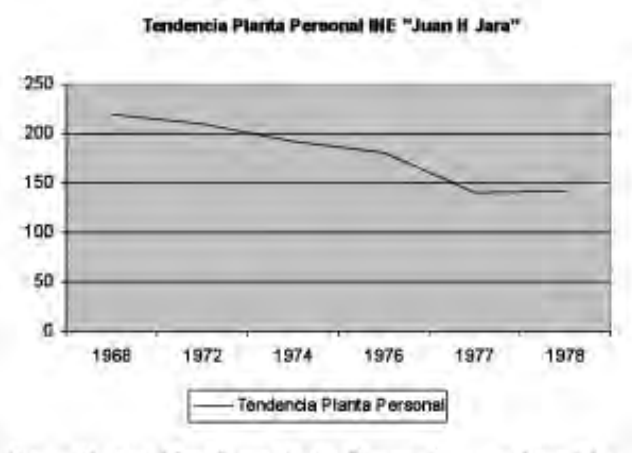

Fuente: Graficu elaborado por el autor. Reoursos humanos disponitilos. Memotias IN.E, (1968-1977).

Para la enfermería, significo la desarticulación y progresiva licuación del capital simbólico que había podido acumular, proceso que no siempre es entendido en todas sus implicancias por los enfermeros, aunque si es asimilado como pérdida: "...este achique de ahora, no es de ahora, ya hubo muchos achiques, y en cada achique se perdió algo, se ganaron cosas y se perdieron" (O.P., entrevista personal, Octubre 2013).

Si bien el modelo de INE tecnocrático había finalmente triunfado, sin embargo el rol previsto para este como formador de políticas de salud nacionales, perdió vigencia junto con el modelo de estado altamente centralizado y la rápida sucesión de gobiernos contrapuestos. A medida que se fueron delegando desde la orbita nacional al ámbito de las provincias y los municipios las tareas más importantes en lo concerniente a la salud, el INE quedo como parte de una estructura obsoleta, un hueso del "gran elefante blanco" que fue alguna vez el Estado nacional.

Podemos ver en las Memorias de los '80s y de los '90s que más allá de algún convenio 
con otras instituciones, el INE no presenta novedades en cuanto a sus funciones ni sus actividades. Solo se va reduciendo lentamente en su planta, con la consiguiente reducción en sus incumbencias.

En enfermería se produjeron algunas incorporaciones a mediados de los 80 's, sin cambiar sustancialmente su status de relativa debilidad dentro de la institución. Un indicio de ello es que el titulo de Licenciatura, que como dice Bourdieu (1985) de los títulos profesionales, es un capital simbólico garantizado social y jurídicamente por el poder de nominación del Estado, no era respetado en la institución por los médicos y las enfermeras tuvieron que librar una lucha permanente para ser incorporadas en la estructura como parte del plantel profesional, lográndolo recién a mediados del 2012.

\section{CONCLUSIONES}

La lucha de la enfermería por encontrar su voz y su visión de la salud, la lucha desigual por construir su capital simbólico propio, se da en un espacio social donde la definición de los principios legítimos de división del campo la tuvieron siempre los médicos. En la visión de éstos, enfermería fue considerado como un "recurso", no muy distinto de otros insumos, que podían construir y deshacer según la necesidad de la coyuntura. Lamentablemente la enfermería en nuestro contexto siempre ha sido muy permeable a esta influencia. Queda planteado para futuras investigaciones averiguar como incide la despolitización generalizada en los ámbitos de la salud publica, y hasta que punto es atribuible al efecto de las constantes interrupciones a la vida democrática en nuestro país.

A los enfermeros les resulta muy difícil situarse por fuera de cierta concepción corporativista y técnico-asistencial de su rol, limitando sus potencialidades para adaptarse a cambios y reacomodarse en escenarios diversos, acotando la posibilidad de realizar un análisis critico ni ofrecer resistencia colectiva a los procesos alienantes. Estas carencias deben ser objeto de preocupación por parte de los que tienen poder de decisión en los ámbitos de formación profesional, es necesario lograr un perfil profesional de enfermería mas crítico y autocrítico.

El caso del INE es paradigmático en este sentido: planteada la necesidad de recortes, las antemencionadas características hicieron de la enfermería el eslabón más débil de la cadena.

En Argentina, la crisis es ya una realidad naturalizada en nuestras instituciones de salud pública, y la provisoriedad y la necesidad se han convertido en regla, dando así por resultado situaciones de desidia y abuso, de desconcierto generalizado, y convirtiendo el desarrollo profesional en una empresa individual que no se vincula con las necesidades históricas de la profesión ni las de la sociedad a la que le debemos nuestros servicios. Es importante "poner en debate y tensión, tramos de la historia de la enfermería argentina" (Morrone, 2012), y también la enfermería universal, para posibilitar la apertura de nuevos horizontes en nuestra profesión.

\section{BIBLIOGRAFÍA}

- Borón, A. (2003) Estado Capitalismo y Democracia en América Latina. CLACSO, Buenos Aires.

- Bourdieu, P. (1985) Espacio social y génesis de las "clases". Espacios, Buenos Aires.

- Bourdieu, P. (1990) Sociología y Cultura. Ed. Grijalbo, Mejico. Disponible en: http://www.armario. cl/2dat/3Apuntes/1Autores/Bourdieu/Bourdieu, P. Sociolog\%EDa y cultura \%5B1984\%5D.pdf

- Iriart, C; Nervi, ; Olivier, B. Y Testa, Mº. (1994) Tecnoburocracia Sanitaria. Lugar Editorial/ Colección Salud Colectiva, Buenos Aires. 
- Colliere, M. (1993) Promover la vida, de la practica de las mujeres cuidadoras a los cuidados de enfermería. Interamericana - Mc Graw Hill, Madrid.

- Diario La Capital, disponible en: http://www.lacapitalmdp.com/noticias/La-Ciudad/2011/12/10/203259.htm

- Giddens, A. (1982) Hermeneútica y teoría social, Capitulo 1 de Profiles and Critiques in Social Theory, University of California Press, 1982.

- Grassi, E. et al, (1994) Politicas Sociales: Crisis y Ajuste Estructural. Ed. Espacio, Buenos Aires.

- Grassi, E. (2004) Política y cultura en la sociedad neoliberal. La otra década infame (II), Espacio Editorial, Buenos Aires

- Lopez, S. (2006) El sistema de Salud Argentino, Material para la materia Medicina Social. Fac. de Trabajo Social - UNLP disponible en: http://www.trabajosocial.unlp. edu.ar/uploads/docs/lopez_el_sistema_de_salud_argentino_.\%20El\%20sistema\%20de\%20salud\%20Argentino.pdf
- Memorias del Instituto Nacional de Epidemiología "Juan H. Jara” 1968-1977. Biblioteca del I.N.E “Juan H Jara”.

- Morrone B. (2012) Redimensionar la historia: Un diálogo provocador con nuestro pasado. Cultura de los Cuidados. (Edición digital) 16, 33. Disponible en: <http:// dx.doi.org/10.7184/cuid.2012.33.01>

- Morrone, B.(2013) Soltando Amarras. Claves para comprender la historia endiente de la enfermería argentina. Ediciones Suárez, 3º Edición. Mar del Plata.

- Padilha, M.I.C.S; Borenstein, M.S. (2005) O metodo de pesquisa histórica na enfermagem. Revista Texto Contexto Enfermagem. 14 (4): 575-84.

- Portantiero, J. C. (1089) La múltiple transformación del Estado Latinoamericano. Nueva sociedad..104: 88-94

- Villalva Beltrán, M. (2012) Sobre hermenéutica: de la Filosofía a la sociología empírica. Revista Española de Sociología. 17:. 9-26

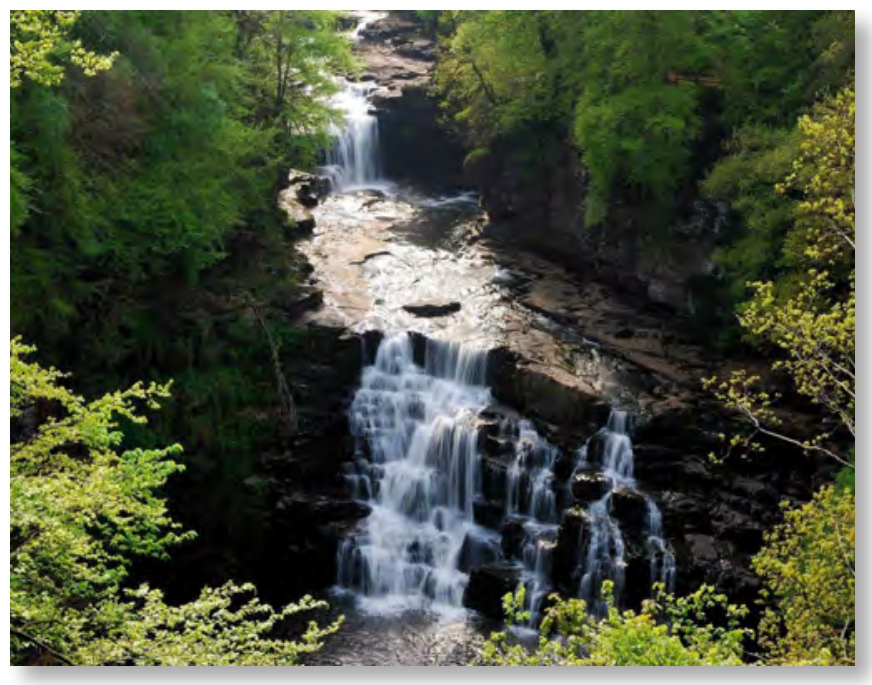

